\title{
Extraction of a computer-certified ODE solver
}

\author{
Grigory Devadze, Lars Flessing, Stefan Streif*
}

\begin{abstract}
Reliably determining system trajectories is essential in many analysis and control design approaches. To this end, an initial value problem has to be usually solved via numerical algorithms which rely on a certain software realization. Because software realizations can be error-prone, proof assistants may be used to verify the underlying mathematical concepts and corresponding algorithms. In this work we present a computercertified formalization of the solution of the initial value problem of ordinary differential equations. The concepts are performed in the framework of constructive analysis and the proofs are written in the Minlog proof system. We show the extraction of a program, which solves an ODE numerically and provide some possible optimization regarding the efficiency. Finally, we provide numerical experiments to demonstrate how programs of a certain high level of abstraction can be obtained efficiently. The presented concepts may also be viewed as a part of preliminary work for the development of formalized nonlinear control theory, hence offering the possibility of computer-assisted controller design and program extraction for the controller implementation.
\end{abstract}

\section{INTRODUCTION}

We consider the initial value problem

$$
\dot{x}=f(t, x), \quad x(0)=x_{0},
$$

where $f: D \rightarrow \mathbb{R}^{n}$ is locally Lipschitz in $x$, continuous in $t$ and is defined on $D \subseteq \mathbb{R}^{+} \times \mathbb{R}^{n}$ with $\left(0, x_{0}\right) \in D$. It is well known that the ordinary differential equations (ODE) do not possess closed-form solutions in general. Thus the initial value problem is an important subject in numerical system analysis and controller design. Usually to apply a numerical method, one would need to predetermine the necessary parameters e.g. precision and step size which may be difficult to obtain beforehand in general. Additionally, pure numerical approximations are not suitable for safety critical applications which require a certain level of rigor and correctness guarantees (see [1] for general IEEE-754 floating point number issues or $[2,3]$ for numerical issues which arise in avionics).

The gap between a mathematical concept and its implementation is sometimes also called algorithmic uncertainty. The importance of deriving (as opposed to just implementing) correct precision-aware controllers within the so-called algorithmic control theory has been highlighted in [4]. Due to the mathematical nature of the problem, computer-assisted formal methods may be used to close this gap and to guarantee correctness fully [5]. In this work we rely on the use of proof assistant software, which have been already successfully applied for important mathematical problems

\footnotetext{
* Technische Universitt Chemnitz,

Automatic Control and System Dynamics Lab, Germany

\{grigory.devadze,lars.flessing,stefan.streif\}@etit.tu-chemnitz.de
}

[6]. Moreover we are interested in the so-called proofsas-programs paradigm to investigate the relation of proofs with computer programming. This procedure is also called program extraction, i.e. a certain proof of the existential theorem corresponds to a computer program which realizes the desired properties correctly. This paradigm can be especially interesting for controller design. Consider e.g., a controller implemented as a software program which has to guarantee a desired property such as stability of the closedloop system. Note that even though development tools such as MATLAB possess certain possibilities for formal code verification [7], this does not guarantee that the implemented controller fulfills the desired properties because the controller itself did not go through a formal analysis. In this work we contribute towards formalized nonlinear control theory with automated program extraction. Please note that related works are reviewed in Section II. As a first step towards this goal, we focus on initial value problems for ODEs, which is undoubtedly a key concept in control theory.

Contributions and outline of this work. The aim of the present work is to provide computer-assisted proofs for the initial value problem (1). As we are interested in the proofas-programs paradigm, we obtain an extracted program, which solves the ODE numerically. Our framework is carried out within a particular realization of exact real arithmetic: constructive analysis. The extracted program is still of a certain high level of abstraction, but can be also run as a usual numerical program.

We start with a review of related work on guaranteed and formal methods in Section II. The employed proof system and the preliminaries of formalization are explained in Section III. The main result, i.e. the formalization of the Cauchy-Euler approximation is derived in Section IV. In Section V we show the application of the derived program with some initial examples and compare the result with existing approaches in proof assistants. In Section 5 we will give an outlook for how these concepts can be further used as a preliminaries for the development of formalized nonlinear control theory.

\section{RELATED WORKS}

To overcome the limitations of missing guarantees in numerical schemes, many different approaches for verified integration have been proposed. [8] presented a method based on local modelling with high-order Taylor polynomials and a bound on the remainder term. [9] utilized the ValEnclIA-IVP to verify whether the enclosure of all reachable states remains within given bounds for known control strategies. However these approaches are still numerical and although the used 
concepts are of a high abstraction level (e.g. Schrauder's fixed point theorem in ([8]), they do not have a formal connection to the actual programming code. In the context of dynamical systems, [10] formally proves the correctness of a program implementing the numerical resolution of a wave equation, with Frama-C ([11]). In [12], the authors formalize variational equations related to the solutions of ordinary differential equations in Isabelle/HOL [13]. With respect to the initial value problem the work of [14] provides the verified implementation of an approximation of a solution of an ODE based on arbitrary precision floating-point numbers. In the present work, the implementation is done by the accurate representation of special infinite sequences of rational numbers. This allows a parametrization of the algorithm by the demanded error bound with the guarantee that this bound holds. Similarly it was done in [15] where the algorithm is derived from the Picard-Lindelöf theorem. However, the level of abstraction of the theorem is very high, such that the running times of the algorithm on even small instances are unusable in practice. In the current work, the level of abstraction is reduced and the approximate solution of the initial value problem is derived efficiently in a direct way by means of the program extraction techniques. Additionally the correctness of the programs follows immediately given from the Soundness Theorem [16].

Proof assistants also play an important role in formal verification of control systems. [17] used Isabelle for implementation of their control system formal verification based on Hybrid Hoare logic. The Why3 platform [18] coupled with MATLAB/Simulink was used in $[19,20]$ to perform simple stability checks of linear discrete systems with quadratic Lyapunov functions. [21] mentioned several proof assistant software tools to be considered for implementation of formal verification of control systems, including [22], HOL-Light [23], Isabelle/HOL and Lean [24]. A closely related work is due to [25] where the authors present the framework for the certified programs for robots based on constructive real numbers [26]. The important work by [27] suggested the first-order formal system for the formalization of hybrid systems, the so-called differential dynamic logic $(\mathrm{dL})$. The key tool which lies in the heart of this formal system is quantifier elimination on the real closed fields [28]. Notably, [29] uses classical formalizations of Picard-Lindelöf theorem to provide soundness results for important $\mathrm{dL}$ axioms: Differential Solution (DS) and Differential Ghost (DG).

\section{Minlog Proof System}

In this section we introduce some preliminary concepts of the proof system Minlog, the notion of totality and the concept of inductive definitions. Afterwards we give the details how the formalization works in constructive setting.

\section{A. Neccessary preliminaries}

In the present work, the analysis and formalization are done within the framework of constructive analysis with a special focus on a concrete and efficient computational realization as well as formal verification.
The interactive proof system Minlog has been developed with the special intention of the program extraction from mathematical proofs. The atomic constructions are done by introducing of free algebras as types which are given by its constructors. The computation is implemented efficiently by the usage of the so-called normalization-by-evaluation. The reason of usage of typed theories is that the normalizationby-evaluation technique, which is applied to the proof terms, needs typed formulas. As an example consider the construction of the type nat of natural numbers $\mathbf{N}$ by stating that Zero is nat and Succ is a mapping of type nat $\Rightarrow$ nat. The normalization of the term $1+2$ would result in Succ(Succ(Succ Zero)).

Program extraction is done from the constructive proofs of theorems. Additionally a proof that an extracted program realizes its specification is machine generated. The user-defined computation and corresponding rewrite rules are assigned to the so-called program constants. Usually the program constants are recursively written typed programs. Consider for example the multiplication of two natural numbers. The corresponding program constant NatTimes is of type nat $\Rightarrow$ nat $\Rightarrow$ nat and the computational rules are given recursively as:

$$
\begin{aligned}
& \text { NatTimes } n \text { Zero } \rightarrow \text { Zero } \\
& \text { NatTimes } n \text { Succ } m \rightarrow \text { NatTimes } n m+n
\end{aligned}
$$

\section{B. Inductive definitions and theorem proving}

Before one starts to prove theorem with the help of the computer, one has to state some definitions which are relevant for the problem statement. In Minlog it is done by the introducing the so-called inductively defined predicate constant (IDPC). Informally speaking, these are formations of implications which leads to the proposition which has to be defined.

As an example assume that one wants to provide a definition for rational numbers which lie in an unit interval. Then the corresponding IDPC RatInUnitI is given by:

$$
\forall a \in \mathbf{Q} 0 \leq a \rightarrow a \leq 1 \rightarrow \text { RatInUnitI } a
$$

which means semantically that to show RatInUnitI $a$ one has to show that $0 \leq a$ and $a \leq 1$.

\section{Preliminaries for the formalization in constructive set- ting.}

This part is based on the foundation of constructive analysis with witnesses [30]. The most important object in the presented setting is a real number. In Minlog reals are build by introducing the typed variables as: $\mathbf{N} \Rightarrow \mathbf{Q}$, $\mathbf{M}: \mathbf{P} \Rightarrow \mathbf{N}$, defining the constructor RealConstr: $(\mathbf{N} \Rightarrow \mathbf{Q}) \Rightarrow$ $(\mathbf{P} \Rightarrow \mathbf{N}) \Rightarrow \mathbf{R}$ and introducing the IDPC Real i.e. the real $x$ is given by a regular Cauchy sequence of rationals $\left(a_{n}\right)_{n}$ with a given weakly increasing modulus $M$.That is $\forall p, m, n\left|a_{n}-a_{m}\right| \leq 2^{-p}$ whenever $M(p) \leq n, m$ and $\forall p, q p \leq q \rightarrow M(p) \leq M(q)$.

Two reals $x=\left(\left(a_{n}\right)_{n}, M\right)$ and $y=\left(\left(b_{n}\right)_{n}, B\right)$ are equal, if $\forall p \in \mathbf{P}\left|a_{M(p+1)}-b_{N(p+1)}\right| \leq 2^{-p}$. A real $x$ is called 
non-negative if $\forall p \in \mathbf{P} 2^{-p} \leq\left(a_{n}\right)_{M(p)}$. For a real $x$ denote $x \in_{p} \mathbf{R}^{+}$(alternately $\left.0<_{p} x\right)$ if $2^{-p} \leq\left(a_{n}\right)_{M(p+1)}$ for some $p$.

Definition 1 (Cont): An uniformly continuous function $f$ : $I \rightarrow \mathbb{R}$ on a compact interval $I$ with rational endpoints is given by

1) an approximating map $h_{f}(I \cap \mathbf{Q}) \times \mathbf{N} \rightarrow \mathbf{Q}$ and a map $\alpha_{f}: \mathbf{P} \rightarrow \mathbf{N}$ such that $\left(h_{f}\left(a_{n}, n\right)\right)_{n}$ is a Cauchy sequence with modulus $\alpha_{f}$

2) a modulus $\omega_{f}: \mathbf{P} \rightarrow \mathbf{P}$ of continuity, which satisfies

$$
|a-b| \leq 2^{-\omega_{f}(p)+1} \rightarrow\left|h_{f}(a, n)-h_{f}(b, n)\right| \leq 2^{p}
$$

with $\alpha_{f}$ and $\omega_{f}$ weakly increasing.

The equivalent realisation of continuous functions in Minlog is done via introducing of the type cont and the IDPC Cont $\mathrm{f}$, stating that $f$ is a proper continuous function. Firstly the basic constructors of cont are:

- $h: \mathbf{Q} \Rightarrow \mathbf{N} \rightarrow \mathbf{Q}$ (approximation mapping)

- $\alpha: \mathbf{P} \Rightarrow \mathbf{N}$ (modulus of convergence)

- $\omega: \mathbf{P} \Rightarrow \mathbf{P}$ (modulus of continuity)

and finally the constructor contConstr: $\mathbf{Q} \Rightarrow \mathbf{Q} \Rightarrow h \Rightarrow$ $\alpha \Rightarrow \omega \Rightarrow$ cont. The property that the mapping of type cont is truly a continuous function in the presented sense can be encoded via the following IDPC cont:

$\forall a 0, b 0, h, \alpha, \omega$ :

$(\forall a(a 0 \leq a \rightarrow a \leq b 0 \rightarrow$ Cauchy $(h a) \alpha)) \rightarrow$

$(\forall a, b, k, n(a 0 \leq a \rightarrow a \leq b 0 \rightarrow a 0 \leq b \rightarrow b \leq b 0 \rightarrow$

$\left.\left.\alpha p \leq n \rightarrow|a-b| \leq 2^{\omega p}\right) \rightarrow|h a-h b| \leq 2^{-p}\right) \rightarrow$

$\forall p, q(p \leq q \rightarrow \alpha p \leq \alpha q) \rightarrow \forall p, q(p \leq q \rightarrow \omega p \leq$ $\omega q) \rightarrow$ Cont (ContConstr aø be $h \alpha \omega$ )

As an example consider the representation of the function $f(x)=x^{2}$ on the interval $[0,2]$ :

ContConstr $02([a, n] a * a)([p] \quad 0) \quad([p] p+3)$

The definition of the vector-valued function is provided similarly:

Definition 2 (ContVec): An uniformly continuous vectorvalued function $\boldsymbol{f}: B \rightarrow \mathbf{R}^{n}$ on a compact ball $B(c, R) \subset$ $\mathbf{R}^{n}$ with rational center $c$ and rational radius $R$ is given by

1) an approximating map $\boldsymbol{h}_{f}\left(B \cap \mathbf{Q}^{n}\right) \times \mathbf{N} \rightarrow \mathbf{Q}$ and a map $\alpha_{f}: \mathbf{P} \rightarrow \mathbf{N}$ such that $\left(h_{f}\left(a_{n}, n\right)\right)_{n}$ is a Cauchy sequence with modulus $\alpha_{f}$

2) a modulus $\omega_{f}: \mathbf{P} \rightarrow \mathbf{P}$ of continuity, which satisfies

$$
\|\boldsymbol{a}-\boldsymbol{b}\|_{1} \leq 2^{-\omega_{f}(p)+1} \rightarrow\left\|\boldsymbol{h}_{f}(\boldsymbol{a}, n)-\boldsymbol{h}_{f}(\boldsymbol{b}, n)\right\|_{1} \leq 2^{p}
$$

with $\alpha_{f}$ and $\omega_{f}$ weakly increasing.

Remark 3: The choice of the norm $\|\cdot\|$ influences the values of moduli $\alpha_{f}$ and $\omega_{f}$. Usually, one would prefer to use either 1 -norm or $\infty$-norm for computational purpose, since for rational vectors the values of the norm are also rationals, which is not the case, e.g., for the Euclidean norm.

Definition 4 (Application): The application of an uniformly continuous function $f: I \rightarrow \mathbb{R}$ to a real $x:=$ $\left.\left(\left(a_{n}\right)_{n}, M\right)\right)$ in $I$ is defined to be a Cauchy sequence $h_{f}\left(a_{n}, n\right)_{n}$ with modulus $\max \left(\alpha_{f}(p+2), M\left(\omega_{f}(p+1)-1\right)\right.$. We denote this real by $f(x)$.
The following theorem is necessary to perform computations with the real numbers.

Theorem 5 (RealApprox): $\forall x, p \exists a\left(|a-x| \leq 2^{-p}\right)$. At this point an easy example of the program extraction in Minlog is provided. Consider the following goal as an equivalent to the theorem statement above:

$$
\forall x, p \text { Real } x \rightarrow \exists \text { a abs }(a-x) \leq(1 \# 2) * * p
$$

After unfolding the definition of $x=\left(\left(a_{n}\right)_{n}, M\right)$ and assuming universally quantified variables $\left(a_{n}\right)_{n}, M$ and the hypothesis Real $x$ the new goal is

$$
\left.\left.\exists \text { a abs(a-(RealConstr }\left(a_{n}\right)_{n} M\right)\right) \leq(1 \# 2) * * \text { p. }
$$

Here the existential quantifier can be eliminated by the term (ex-intro (pt " $\left(a_{n}\right)_{M(p)}$ "). The rest of the proof uses usual techniques to prove that the $\left|\left(a_{n}\right)_{M(p)}-\left(\left(a_{n}\right)_{n}, M\right)\right| \leq 2^{p}$. Once the existential proof of the theorem is done, this theorem can be animated. In case of the animation of RealApprox one obtains a program constant with the following computation rule:

cRealApprox $\rightarrow[\mathrm{x} \theta, \mathrm{p} 1]$

[if $x 0([a s 2, M 3] \operatorname{as} 2(M 3$ p1) )] which is an extracted program from the constructive proof of the theorem RealApprox. Moreover, one can use the extracted program in the proof of the following theorem and generate new program:

Theorem 6 (RealVecApprox): $\forall \boldsymbol{x} \in \mathbf{R}^{n}, p \exists \boldsymbol{e} \in \mathbf{Q}^{n} \| \boldsymbol{e}-$ $\boldsymbol{x} \|_{1} \leq 2^{-p}$.

Proof: Proceed by induction on n. For $n=1$ the existence holds by the theorem RealApprox and is realized by cRealApprox. For $n=2$ and for any vector real number $\boldsymbol{x}=\left(x_{1}, x_{2}\right)$ generate a vector $\boldsymbol{e}=\left(e_{1}, e_{2}\right)$ with $\mid e_{1}-$ $x_{1}\left|\leq 2^{-(p+1)},\right| e_{2}-x_{2} \mid \leq 2^{-(p+1)}$ again by the Theorem RealApprox. Clearly $\left|e_{1}-x_{1}\right|+\left|e_{2}-x_{2}\right| \leq 2^{-p}$. For the induction step assume that for the vector $\boldsymbol{x}^{\prime}=\left(x_{1}^{\prime}, \ldots, x_{n}^{\prime}\right)$ there exists a vector $\boldsymbol{e}^{\prime}$ with $\left\|\boldsymbol{e}^{\prime}-\boldsymbol{x}^{\prime}\right\| \leq 2^{-(p+1)}$ and for the real number $x_{n+1}$ there exists a rational number $e_{n+1}$ again by RealApprox such that $\left|e_{n+1}-x_{n+1}\right| \leq 2^{-(p+1)}$. Then for the vector $\boldsymbol{x}=\left(x_{1}, \ldots, x_{n+1}\right)$ the vector $e=\left(e^{\prime}, \ldots, e_{n+1}\right)$ satisfies the desired condition and is a $2^{-p}$ approximation to a real vector $x$.

Thus we are able to perform correct computations with vector-valued functions, since the program constant Application is capable to generate new real number vectors $\boldsymbol{f}(\boldsymbol{x})$ correctly.

Definition 7 (Time derivative): Let $\varphi_{1}:\left[T_{1}, T_{2}\right] \rightarrow$ $\mathbf{R}^{n}, \boldsymbol{\varphi}_{2}:\left[T_{1}, T_{2}\right] \times B \rightarrow \mathbf{R}^{n}$ be continuous in sense of Definition 2 and $0 \leq T_{1}<T_{2}$. The function $\varphi_{2}$ is called partial derivative w.r.t. the time of $\varphi_{1}$ with the modulus of differentiability $\delta_{\varphi_{1}}: \mathbf{P} \rightarrow \mathbf{P}$ if for $t_{1}, t_{2} \in\left[T_{1}, T_{2}\right]$ with $t_{1}<t_{2}$,

$$
\begin{aligned}
& \forall p t_{2} \leq t_{1}+2^{-\delta_{\boldsymbol{\varphi}_{1}(p)}} \rightarrow \\
& \left|\boldsymbol{\varphi}_{1}\left(t_{2}\right)-\boldsymbol{\varphi}_{1}\left(t_{1}\right)-\boldsymbol{\varphi}_{2}\left(t_{1}, \boldsymbol{x}\right)\left(t_{2}-t_{1}\right)\right| \leq 2^{-p}\left(t_{2}-t_{1}\right)
\end{aligned}
$$

We denote the function $\varphi_{2}$ as $\dot{\varphi}_{1}$ and call the function $\varphi_{1}$ differentiable on $\left[T_{1}, T_{2}\right]$.

The next steps allow to generate a uniform partition of a rational interval such that the functions can be evaluated at 
discrete values.

Definition 8 (Partition): Let $a, b$ be rational numbers such that $a<b$. A list $P=a_{0}, \ldots, a_{n}$ of rationals is a partition of the interval $[a, b]$, if $a=a_{0} \leq a_{1} \leq \cdots \leq a_{n}=$ $b$.

Theorem 9 (RatLeAbsBound): $\forall a \in \mathbf{Q} \exists p \in \mathbf{P}|a| \leq 2^{p}$. The corresponding extracted program of type rat $\Rightarrow$ pos is denoted as cRatLeAbsBound.

Definition 10: The program constant UnifP of type nat $\Rightarrow$ rat $\Rightarrow$ rat $\Rightarrow$ (list rat) is recursively defined as follows:

- UnifP Zero $a b \rightarrow b$ :

- UnifP (Succ n) a b $\rightarrow$

UnifP (n) a $((a+b) / 2)$ :+: UnifP (n) a $((a+b) / 2)$

where $:+$ : is list concatenation.

Definition 11: The program constant UnifP of type rat $\Rightarrow$ pos $\Rightarrow$ (list rat) is defined as:

$(\theta)$ : : UnifP Succ ( $p+c$ RatLeAbsBoundPos a) 0 a

The direct statement of the goal

$$
\forall b, p \text { Partition } 0 b 2^{-p} \text { UnifP b } \mathrm{p}
$$

corresponds to the statement of the following theorem. Thus we are able to prove that the program constant UnifP indeed generates a list which is Partition.

Theorem 12 (UnifPPartition): For $a \in \mathbf{Q}, p \in \mathbf{P}$ the list of rationals generated by the program constant UnifP is a partition $P=c_{0}, \ldots, c_{n}$ of the interval $[0, a]$. Additionally it holds that $\max \left\{c_{i+1}-c_{i} \mid i<n\right\} \leq 2^{-p}$.

Definition 13 (EulerMap): The program constant EulerMap of type contVec $=>$ ratVec $=>$ nat $=>$ rat $=>$ pos $=>$ ratVec is recursively defined as follows:

- EulerMap $\boldsymbol{f} \boldsymbol{a}$ Zero d $\mathrm{p} \rightarrow \boldsymbol{a}$

- EulerMap $f \boldsymbol{a}$ (Succ n) d p $\rightarrow$ EulerMap $\boldsymbol{f} \boldsymbol{a}$ n d p $+d \cdot$ cRealVecApprox $(f(n * d$, EulerMap $f \boldsymbol{a}$ n $d p)) p$

In summary, in this section we presented the most important concepts of the Minlog system and presented the formalization of constructive analysis in Minlog as well as the extension of the standard library which was necessary for our goal. In the next section we provide the formalization of Cauchy-Euler approximation method in our framework.

\section{MAin Result}

\section{A. Formalized proof in Minlog}

Using the machinery presented in Section III we prove the existence of an approximate solution by the means of the Cauchy-Euler approximation method. We consider a differential equation (properly stated in our constructive framework):

$$
\dot{\boldsymbol{x}}=\boldsymbol{f}(t, \boldsymbol{x}), \quad \boldsymbol{x}(0)=\boldsymbol{x}_{0} .
$$

Although the proof is standard, we provide it here in formalized way which is very close to a computer proof.

Theorem 14 (CauchyEulerApproxSolCorr): Let $f: B \rightarrow$ $\mathbf{R}^{n}$ with $B(c, R) \subset \mathbf{Q}^{n}$ be an uniformly continuous vectorvalued function and $\left(0, \boldsymbol{x}_{0}\right) \in B$. Assume that for the set $B^{\prime}$ given by $|t| \leq t_{a},\left\|\boldsymbol{x}-\boldsymbol{x}_{\mathbf{0}}\right\|_{1} \leq x_{b}$ is in $B$. Assume there exists a rational number $C>0$ such that $\|\boldsymbol{f}(t, \boldsymbol{x})\|_{1} \leq C$ for $(t, \boldsymbol{x}) \in B^{\prime}$. Then for every $p \in \mathbf{P}$ there exist an approximate solution $\varphi:[0, T] \rightarrow \mathbf{Q}^{n}$ up to the error $2^{-p}$ in sense that

$$
\|\dot{\boldsymbol{\varphi}}(t)-\boldsymbol{f}(t, \boldsymbol{\varphi}(t))\|_{1} \leq 2^{-p}
$$

for all $t \in[0, T]$ with $T=\min \left\{t_{a}, \frac{x_{b}}{M}\right\}$ where $\dot{\varphi}(t)$ is defined.

Proof: For any $p \in \mathbf{P}$ obtain a number $q \in \mathbf{P}$ by the continuity such that:

$$
\left\|f\left(t_{1}, \boldsymbol{x}\right)-f\left(t_{2}, \boldsymbol{y}\right)\right\|_{1} \leq 2^{-(p+1)},
$$

whenever $\|\boldsymbol{x}-\boldsymbol{y}\|_{1},\left|t_{1}-t_{2}\right| \leq 2^{-q}$. Generate a partition $P=c_{0}, \ldots, c_{n}$ by applying the program constant UnifP of the interval $[0, T]$ such that $\max \left\{c_{i+1}-c_{i} \mid i<n\right\} \leq$ $\min \left(2^{-q}, 2^{-(q+\text { cRatLeAbsBound } C)}\right)$.

Recursively construct the approximate solution $\varphi$ via the program constant EulerMap. Note that the approximate solution satisfies for $i=1, \ldots, n$ :

$$
\begin{aligned}
& \boldsymbol{\varphi}(t)=\boldsymbol{b}_{i}+\left(t-c_{i}\right) \boldsymbol{s}_{i-1} \text { for } c_{i-1} \leq t \leq c_{i} \\
& \boldsymbol{b}_{i}=\boldsymbol{\varphi}\left(c_{i}\right),
\end{aligned}
$$

where $s_{i-1}$ are calculated such that $\left\|s_{i}\right\|_{1} \leq C$ and $\left\|s_{i-1}-f\left(\boldsymbol{b}_{i}, c_{i}\right)\right\| \leq 2^{-(p+1)}$ by the program constant cRealVecApprox.

Observe that for $c_{i-1}<t<c_{i}$

$$
\begin{aligned}
& \left\|\boldsymbol{\varphi}(t)-\boldsymbol{b}_{i-1}\right\|_{1} \leq\left|t-c_{i-1}\right|\left\|s_{i-1}\right\|_{1} \\
& \leq 2^{-(q+\text { cRatLeAbsBound } C)} 2^{\text {cRatLeAbsBound } C)} \\
& \leq 2^{-q}
\end{aligned}
$$

and finally,

$$
\begin{aligned}
& \| \dot{\boldsymbol{\varphi}}(t)-f\left(t, \boldsymbol{\varphi}(t)\left\|_{1}=\right\| s_{i-1}-f(t, \boldsymbol{\varphi}(t)) \|_{1}\right. \\
& \leq\left\|s_{i-1}-f\left(c_{i}, \boldsymbol{b}_{i}\right)\right\|_{1}+\| f\left(c_{i}, \boldsymbol{b}_{i}\right)-f\left(t, \boldsymbol{\varphi}(t) \|_{1}\right. \\
& \leq 2^{-(p+1)}+2^{-(p+1)} \leq 2^{-p} .
\end{aligned}
$$

Remark 15: For existence and uniqueness we rely on the pen-and-paper version [31] and admit its correctness for now. Let $f: B \rightarrow \mathbb{R}$ be continuous and satisfy the Lipshitz condition w.r.t. its second argument, i.e. there exists a constant $L$ such that $\left.\| f\left(t, \mathbf{x}_{1}\right)-\mathbf{x}_{2}\right)\|\leq L\| \mathbf{x}_{1}-\mathbf{x}_{2} \|$ for any $x_{1}, x_{2} \in B$. Assume that the assumption of the Theorem 14 holds. Then we can construct a unique exact solution $\boldsymbol{\psi}:[0, T] \rightarrow \mathbb{R}^{n}$ to (2) such that $\boldsymbol{\psi}(0)=\boldsymbol{x}_{0}$. Moreover let $\phi:[0, T] \rightarrow \mathbb{R}^{n}$ be an approximate solution to (2). Then

$$
\forall p, t \in[0, T]\|\varphi(t)-\psi(t)\|_{1} \leq \frac{2^{-p}}{L}\left(e^{L \cdot T-1}\right) .
$$

Thus proper evaluation of approximate solution $\varphi$ yields arbitrary approximation of an exact solution of (2).

Remark 16: The extracted program $\varphi$ is of type contvec and fulfills the assumptions of Definition 2 (ContVec). The respective modulus of continuity $\omega$ and modulus of convergence $\alpha$ are derived by the proof system automatically. Moreover $\varphi(t)$ is admissible, i.e. $(t, \varphi(t)) \in B$ and piecewise continuous differentiable. 


\section{B. Program extraction}

The direct extraction yields the invocation of the program constant EulerMap, which is not usable for practical computation. Thus we define new program constant EulerMapFast of the type contVec $=>($ ratVec pair ratVec $)=>$ rat $=>$ pos $=>($ ratVec pair ratVec) recursively as:

- EulerMapFast Zero rt d p $\rightarrow$ rt

- EulerMapFast (Succ n) rt d p $\rightarrow$ EulerMapFast n ((rht $r t)$ pair

(rht $r t+d * c$ RealVecApprox $f(n * d,($ rht rt $)) \quad p) c p$

where pair is a constructor for a tuple, $r t$ is a varible of type (ratVec pair ratVec) and rht accesses the right element of the tuple.

Next we showed the equivalence of outputs of the program constants EulerMap $\boldsymbol{f} \boldsymbol{a} \mathrm{n}$ d $\mathrm{p}$ and EulerMapFast $\mathrm{n}$ (Inhab rat) pair $\boldsymbol{a} \mathrm{d}$, where (Inhab ratVec) is an arbitrary inhabitant of rational vectors. Observe that EulerMapFast mimics a usual for loop and there are no more redundant recursive calls as in EulerMap. However EulerMap yields easier goals for the reasoning i.e. we use EulerMapFast for a computational version of the Theorem 14 where the program equivalence guarantees the correctness. Additionally we evaluate all elementary operations $(+,-, \cdot, /$, floor $)$ on rational numbers externally in scheme due to the fact that the operations which are defined for decidable/discrete predicates (rational numbers in this case) should be not affected by some algorithmic uncertainty. Furthermore, scheme cancels the greatest common divisor automatically, thus we do not have to be concerned about it during the theorem proving. As for the last optimization we implemented the so-called compression of real numbers. That is, for any real $x=\left(\left(a_{n}\right)_{n}, M\right)$ there is a new real $y=\left(\left(b_{n}\right)_{n}, p+2\right)$ with $\left(b_{n}\right)_{n}:=\frac{\text { floor }\left(a_{M(p)} \cdot 2^{n}\right)}{2^{n}}$ with $x=y$. This allows us to control the length of the denominator and makes the Cauchy sequences less complex. Applying this optimization allows performing the computation more efficiently which is shown experimentally in the next section.

\section{CASe Study}

In the following we compare our results with the existing approaches in proof assistants on the following basic but frequently used examples in this line of research $[15,8]$.

Example 1:

For the differential equation

$$
\dot{x}=x, x(0)=1
$$

the data required for the application of Theorem 14 is:

- $h_{f}\left(a_{1}, a_{2}, n\right)=a_{1}$,

- $\alpha(p)=0$,

- $\omega(p)=p$.

- $C=2, L=1$.

We obtain two correct decimal digits $(p=10)$ at $t=\frac{1}{2}$ i.e. the result is the rational number $\frac{55317227}{33554432}$ within $3 \mathrm{sec}$, whereas Coq C-CORN needs approximately $10 \mathrm{~min}$. As for the work of [14], the timing is comparable, however, we did not need to consider the notion of the stability of the Euler method, since our approach runs an "ideal" Euler method efficiently. Moreover, we stay within Minlog's verification kernel except for some basic computations on rationals.

Example 2: Integrating the circle

$$
\begin{array}{rr}
\dot{x}=-y, & x(0)=1 \\
\dot{y}=x, & y(0)=0
\end{array}
$$

We use the following representation:

- $\boldsymbol{h}_{\boldsymbol{f}}\left(a_{1}, a_{2}, a_{3}, n\right)=\left(-a_{2}, a_{1}\right)$,

- $\alpha(p)=0$,

- $\omega(p)=p$,

- $C=2, L=1$

For two correct decimal digits of the integration from 0 to $2 \pi$ the resulting rational vector is $\left(\frac{16790121}{16777216}, \frac{22267}{134217728}\right)$ and its timing is approximately $30 \mathrm{~min}$ which corresponds to approximately 26000 verified evaluations of $f$. Note that our assumptions are valid for any $t$, thus we are allowed to run the algorithm successively and obtain the continuation of the solution.

Overall we conclude that our extracted program achieved relatively good performance. Additionally, observe that we are able to obtain a verified result at any desired precision in principle. Please note that extracted programs in Minlog allow the evaluation of verified high-level functions directly. Clearly the procedure of program extraction is not limited to the optimization presented in Section IV and the running time can be further reduced drastically. A detailed analysis is beyond the scope of this work and subject of current investigations. Note further that while the analysis of trajectories is often essential for the controller design and its formal verification, the controller acting online may be much simpler and may not need to determine the trajectory.

\section{CONCLUSION}

We formalized the Cauchy-Euler approximation method for the solution of the initial value problem for the ordinary differential equation in Minlog. We performed the program extraction in the exact real number arithmetic and showed how the extracted program can be optimized in terms of efficiency and maintaining a certain high level of abstraction. The presented results might be fruitful for the reasoning on higher-order numerical schemes like Runge-Kutta. Furthermore the notion of system trajectory plays a crucial role in the stability of the equilibrium of nonlinear systems where the notion of uniform asymptotic stability is of a separate interest, due to the arising of special initial value problem from the comparison principle [32]. Thus the presented concepts may also be viewed as a part of preliminary work for the development of formalized nonlinear control theory.

\section{REFERENCES}

[1] B. A. Wichmann, "A note on the use of floating point in critical systems," The Computer Journal, vol. 35, no. 1, pp. 41-44, 1992.

[2] D. Delmas, E. Goubault, S. Putot, J. Souyris, K. Tekkal, and F. Védrine, "Towards an industrial use of fluctuat on safety-critical avionics software," in International 
Workshop on Formal Methods for Industrial Critical Systems. Springer, 2009, pp. 53-69.

[3] A. E. Goodloe, C. Muñoz, F. Kirchner, and L. Correnson, "Verification of numerical programs: From real numbers to floating point numbers," in NASA Formal Methods Symposium. Springer, 2013, pp. 441-446.

[4] P. Tsiotras and M. Mesbahi, "Toward an algorithmic control theory," Journal of Guidance, Control, and Dynamics, vol. 40, no. 2, pp. 194-196, 2017.

[5] O. Hasan and S. Tahar, "Formal verification methods," in Encyclopedia of Information Science and Technology, Third Edition. IGI Global, 2015, pp. 7162-7170.

[6] G. Gonthier, A. Asperti, J. Avigad, Y. Bertot, C. Cohen, F. Garillot, S. Le Roux, A. Mahboubi, R. O'Connor, S. O. Biha et al., "A machine-checked proof of the odd order theorem," in International Conference on Interactive Theorem Proving. Springer, 2013, pp. 163179.

[7] C.-C. Lee and J. Friedman, "Requirements modeling and automated requirements-based test generation," SAE International Journal of Aerospace, vol. 6, no. 2013-01-2237, pp. 607-615, 2013.

[8] M. Berz and K. Makino, Verified Integration of ODEs and Flows Using Differential Algebraic Methods on High-Order Taylor Models. Kulwer Academic Publishers, 1998, pp. 361-369.

[9] A. Rauh and H. Aschemann, Interval Methods for Verification and Implementation of Robust Controllers. Berlin, Heidelberg: Springer Berlin Heidelberg, 2011, pp. 201-211.

[10] S. Boldo, F. Clément, J.-C. Filliâtre, M. Mayero, G. Melquiond, and P. Weis, "Wave equation numerical resolution: a comprehensive mechanized proof of a c program," Journal of Automated Reasoning, vol. 50, no. 4, pp. 423-456, 2013.

[11] P. Cuoq, F. Kirchner, N. Kosmatov, V. Prevosto, J. Signoles, and B. Yakobowski, "Frama-c," in International Conference on Software Engineering and Formal Methods. Springer, 2012, pp. 233-247.

[12] F. Immler and C. Traut, "The flow of ODEs: Formalization of variational equation and Poincaré map," Journal of Automated Reasoning, vol. 62, no. 2, pp. 215-236, 2019.

[13] T. Nipkow, L. Paulson, and M. Wenzel, Isabelle/HOL A Proof Assistant for Higher-Order Logic, ser. LNCS. Springer, 2002, vol. 2283.

[14] F. Immler, "A verified ODE solver and the Lorenz attractor," Journal of Automated Reasoning, vol. 61, no. 1-4, pp. 73-111, 2018.

[15] E. Makarov and B. Spitters, "The Picard algorithm for ordinary differential equations in Coq," in International Conference on Interactive Theorem Proving. Springer, 2013, pp. 463-468.

[16] U. Berger, K. Miyamoto, H. Schwichtenberg, and M. Seisenberger, "Minlog - a tool for program extraction supporting algebras and coalgebras," in International Conference on Algebra and Coalgebra in
Computer Science. Springer, 2011, pp. 393-399.

[17] L. Zou, J. Lv, S. Wang, N. Zhan, T. Tang, L. Yuan, and Y. Liu, "Verifying chinese train control system under a combined scenario by theorem proving," in Proc. Working Conf. Verified Software: Theories, Tools, and Experiments. Springer, 2013, pp. 262-280.

[18] F. Bobot, J.-C. Filliâtre, C. Marché, G. Melquiond, and A. Paskevich, "The why3 platform," LRI, CNRS \& Univ. Paris-Sud \& INRIA Saclay, version 0.64 edition, 2011.

[19] D. Araiza-Illan, K. Eder, and A. Richards, "Formal verification of control systems' properties with theorem proving," in Proc. 2014 UKACC Int. Conf. Control, 2014, pp. 244-249.

[20] - "Verification of control systems implemented in simulink with assertion checks and theorem proving: A case study," in Proc. 2015 European Control Conf. (ECC), 2015, pp. 2670-2675.

[21] S. Gao, "Descriptive control theory: A proposal," arXiv preprint arXiv:1409.3560, 2014.

[22] Coq, "The Coq proof assistant reference manual," 2004, Version 8.0. [Online]. Available: http://coq.inria.fr

[23] J. Harrison, "HOL Light: A tutorial introduction," in International Conference on Formal Methods in Computer-Aided Design. Springer, 1996, pp. 265-269.

[24] L. de Moura, S. Kong, J. Avigad, F. Van Doorn, and J. von Raumer, "The lean theorem prover (system description)," in International Conference on Automated Deduction. Springer, 2015, pp. 378-388.

[25] A. Anand and R. Knepper, "ROSCoq: Robots powered by constructive reals," in International Conference on Interactive Theorem Proving. Springer, 2015, pp. 3450.

[26] R. O'Connor, "Certified exact transcendental real number computation in Coq," in International Conference on Theorem Proving in Higher Order Logics. Springer, 2008, pp. 246-261.

[27] A. Platzer, "Logics of dynamical systems," in Proc. 2012 IEEE/ACM Symp. Logic Computer Science (LICS). IEEE Computer Society, 2012, pp. 13-24.

[28] A. Tarski, "A decision method for elementary algebra and geometry," in Quantifier Elimination and Cylindrical Algebraic Decomposition. Springer, 1998, pp. 2484.

[29] B. Bohrer, V. Rahli, I. Vukotic, M. Völp, and A. Platzer, "Formally verified differential dynamic logic," in Proceedings of the 6th ACM SIGPLAN Conference on Certified Programs and Proofs, ser. CPP 2017. New York, NY, USA: Association for Computing Machinery, 2017, p. 208-221.

[30] H. Schwichtenberg, "Constructive analysis with witnesses," Proof Technology and Computation. Natio Science Series, pp. 323-354, 2006.

[31] W. Hurewicz, Lectures on ordinary differential equations. Courier Corporation, 1990.

[32] H. Khalil, Nonlinear Systems. Prentice-Hall. 2nd edition, 1996, pp. 139-140. 